\title{
Effects of Ultraviolet Intensity and Initial Thickness on Normal Force of Acrylic Ultraviolet Curable Resin
}

\author{
Kentaro Taki ${ }^{* *}$ and Keigo Sawa ${ }^{2}$ \\ ${ }^{\text {IS }}$ chool of Mechanical Engineering, \\ ${ }^{2}$ Department of Natural System, Graduate School of Natural Science, \\ Kanazawa University, Kakuma-machi, Kanazawa, Ishikawa, 920-1192, Japan \\ *taki@se.kanazawa-u.ac.jp
}

\begin{abstract}
Measuring residual stress is important for evaluating the long-term quality of assembled products that feature ultraviolet (UV) curable resin as an adhesive. In this study, the residual stress of UV-curable adhesive was evaluated by measuring the normal force acting on UVcured resin filled between two plates, using a photo-rheometer. The normal force was generated rapidly when the UV curable resin was exposed to UV light. The change in the normal force then became more gradual. Increasing the UV intensity and thickness increased the normal force. The onset time of normal force generation became faster for thinner initial thicknesses and high UV intensity. There were positive correlations between the curing speed and UV intensity, as well as between the residual normal force and gap.
\end{abstract}

Keywords: Normal force, Residual stress, Photo-rheometer, Photopolymerization, Adhesive

\section{Introduction}

Ultraviolet (UV)-curable adhesive is widely used in the electronics, high-precision optical equipment, and automobile industries, among others. UVcurable resin produces residual force between two fixed surfaces, and can shrink if the resin has a free surface during photopolymerization. Both residual force and shrinking are unavoidable aspects of UVcurable resin.

Residual force and shrinking behavior have both been measured using various apparatus. A linometer attached to a UV light has been utilized to measure volumetric contraction and evaluate shrinkage behavior [1]. A device with a cantilever beam bending principle has been used to monitor shrinkage stress $[2,3]$. Photo-rheometers have also been used to measure UV-curing kinetics in the past quarter of a century [4]. Real-time near infrared spectroscopy, equipped with a photo-rheometer (as developed by Liska's group), has advanced the kinetic study of normal force (shrinkage force) and double bond conversion [5-11]. A laser displacement sensor has been used in a controlled atmosphere to elucidate the degree of shrinkage, both in air and oxygen-free atmospheres [12].

In the field of nanoimprint lithography (NIL) technology, understanding the shrinkage phenomenon is crucial for ensuring the accuracy of the final product. Laser displacement sensors have also been used to measure the kinetics of shrinkage during UV-curing [13]. A detaching force from a NIL mold, which may be related to the residual force, has also been measured using a device where a force sensor was attached to the NIL mold [14]. The numerical simulation of the NIL process, taking shrinkage into account, has also been developed by Hirai's group [15-18] and another group [19]. Moreover, in the fields of additive manufacturing or three-dimensional (3D) printing using UV-curable resins, residual stress, and shrinkage are major concerns [20].

Though many studies have focused on the measurement of shrinking kinetics and residual force, most of these studies have employed special apparatus. A commercially available and facile measurement technique has not yet been established. 
Moreover, a practical correlation that can be used for a finite element analysis (FEA) of computeraided engineering (CAE) is still insufficient.

In this study, an option for a commercially available photo-rheometer was utilized to measure the normal force of UV-curable resin confined between two plates, to evaluate the kinetics of its residual force. The change in normal force was monitored over time. The effects of UV intensity and gap width were evaluated, and the correlations were proposed.

\section{Experimental}

\subsection{Materials}

A commercially available acrylic UV-curable adhesive (Epo-TEK ${ }^{\circledR}$ OG603, Epoxy Technology) was supplied, and was used without further purification.

\subsection{UV light source}

A high-pressure mercury lamp (OmniCure S2000, ExFo, Canada) was used as the light source, and UV intensity was measured using a photometer (UIT150 , Ushio, Japan) with a maximum sensitivity at $365 \mathrm{~nm}$.

\subsection{Photo-rheometer}

A photo-rheometer (MCR-302-WESP) was used with the Peltier temperature control units (PPTD200/GL and H-PTD200). The photo-rheometer setup was modified slightly. One of the advantages of using a photo-rheometer is that it can set a gap with an accuracy of $\pm 1 \mu \mathrm{m}$. The gap is important for realizing high-reproducibility, as the UV light is attenuated in the sample.

As shown in Fig. 1, the photo-rheometer consists of the UV light source, two fused silica windows, a circular disk with a hole, a $\phi 12-\mathrm{mm}$ fixture, and a hood. We modified the commercial setup as follows: the fixture and the circular disk were stained black via treatment with alumite to avoid any of the UV light straying. The circular disk was essential, as it enhanced thermal conduction and made the temperature distribution uniform in the fused silica windows. The position of the UV light was adjusted to align the optical axis of the UV light to the axis of the fixture, using the xyz stage attached to the UV light guide. By altering the horizontal position of the UV light using the xy stage, the UV intensity at the axis of the fixture was brought to its maximum. Then, the UV intensity was adjusted to the desired UV intensity by translating the UV light position and changing the iris of the
UV lamp within $0.1 \mathrm{~mW} / \mathrm{cm}^{2}$. Measurement was started $20 \mathrm{~min}$ after sample loading, to ensure that there was a temperature equilibrium. These modifications and devices improved the reproducibility of measurement dramatically.

Measurements were conducted at $30^{\circ} \mathrm{C}$. The gap was changed from 0.04 to $0.9 \mathrm{~mm}$. The UV intensity was measured at $1.46,5$, and $29 \mathrm{~mW} / \mathrm{cm}^{2}$. The irradiation time was $30 \mathrm{~s}$. The measurement period and rotational frequency were $0.1 \mathrm{~s}$ and $10 \mathrm{~Hz}$, respectively. The normal force was monitored for 90 $\mathrm{s}$, including the irradiation time.

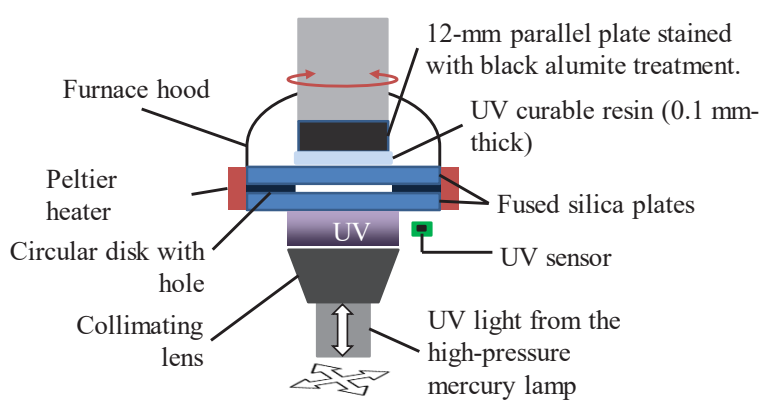

Fig. 1. Schematic of the photo-rheometer setup.

\section{Results and discussion}

The changes from the normal position for each UV intensity are shown in Figs. 2 to 4. In Fig. 2, the normal force started to increase at $2 \mathrm{~s}$, and increased up to $7.2 \mathrm{~N}$ for a $0.04 \mathrm{~mm}$ gap. For a $0.5 \mathrm{~mm}$ gap, the normal force increased from $11.1 \mathrm{~s}$, and achieved a maximum of $25 \mathrm{~N}$. The onset time was defined as the initial slope of the normal force curve, as shown in Fig. 2. There is a clear trend in that the thinner the gap was, the more the onset time was reduced, and the lower the normal force at $90 \mathrm{~s}$ was.

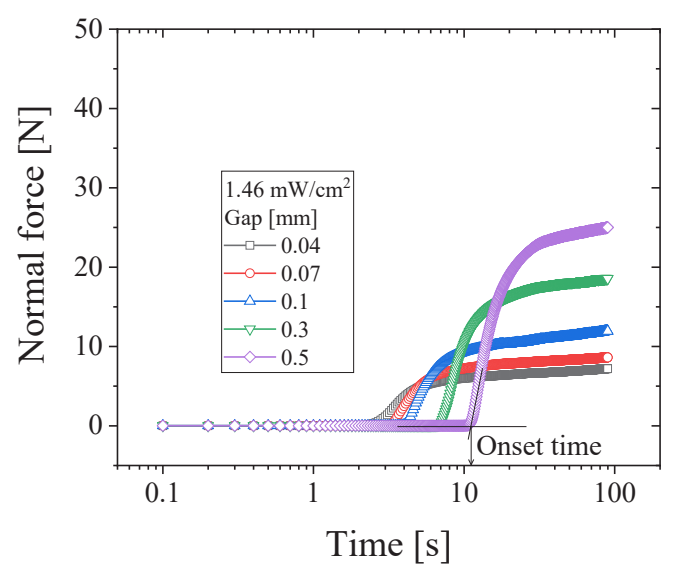

Fig. 2. Effect of gap width on the change in normal force $\left(1.46 \mathrm{~mW} / \mathrm{cm}^{2}\right)$. 
Figure 3 shows the normal force curves at 5 $\mathrm{mW} / \mathrm{cm}^{2}$. The onset time of the $0.04 \mathrm{~mm}$ gap was 1 $\mathrm{s}$, which was faster than that at $1.46 \mathrm{~mW} / \mathrm{cm}^{2}$. The normal force at $90 \mathrm{~s}$ was $7.9 \mathrm{~N}$, which was larger than that at $1.46 \mathrm{~mW} / \mathrm{cm}^{2}$. This tendency was the same for other gap widths.

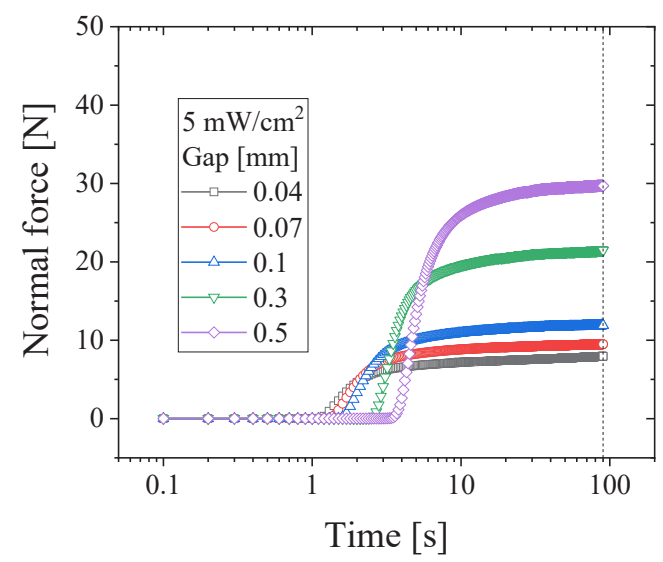

Fig. 3. Effect of gap width on the change in normal force $\left(5 \mathrm{~mW} / \mathrm{cm}^{2}\right)$.

The normal force curves at $29 \mathrm{~mW} / \mathrm{cm}^{2}$ are shown in Fig. 4. The general trend of onset time and the normal force at $90 \mathrm{~s}$ were the same as other at the UV intensities. Compared to other UV intensities, the experiment performed at $29 \mathrm{~mW} / \mathrm{cm}^{2}$, which was the highest UV intensity among those examined, exhibited the fastest onset time and the highest normal force at $90 \mathrm{~s}$ for each gap width.

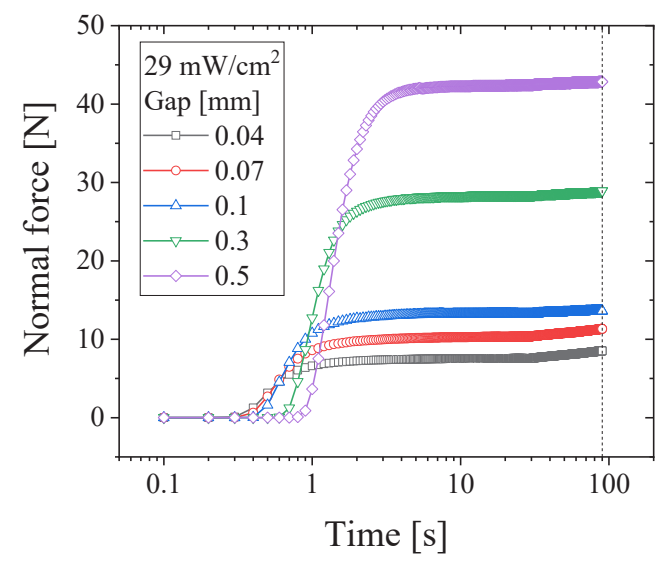

Fig. 4. Effect of gap width on the change in normal force $\left(29 \mathrm{~mW} / \mathrm{cm}^{2}\right)$.

Figure 5 shows the relationships between the onset time, gap width, and UV intensity. The plots and error bars show the experimental results, and the straight lines show linear correlations. The onset time is a linear function of the film thickness. The slopes of the lines increase with decreasing UV intensity. This indicates that the onset time increased for large film thicknesses, and under low UV intensity. The slopes and intercepts of the linear correlation lines are summarized in Table 1.

The inverse of the slope is defined as the curing speed, $v_{\mathrm{c}}$, with units of $\mathrm{mm} / \mathrm{s}$ expressed as Eq. (1):

$$
v_{\mathrm{c}}=\frac{1}{a}
$$

where $a$ is the slope of the onset time to the gap width. The curing speed of each UV intensity is plotted on a log-log scale in Fig. 6. The curing speed shows a scaling relationship, and its factor is 1.41.

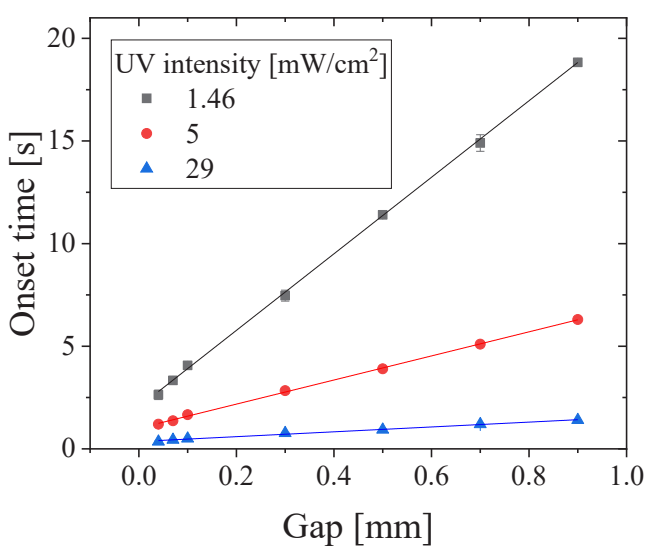

Fig. 5. Relationships between the onset of normal force increase, gap width, and UV intensity.

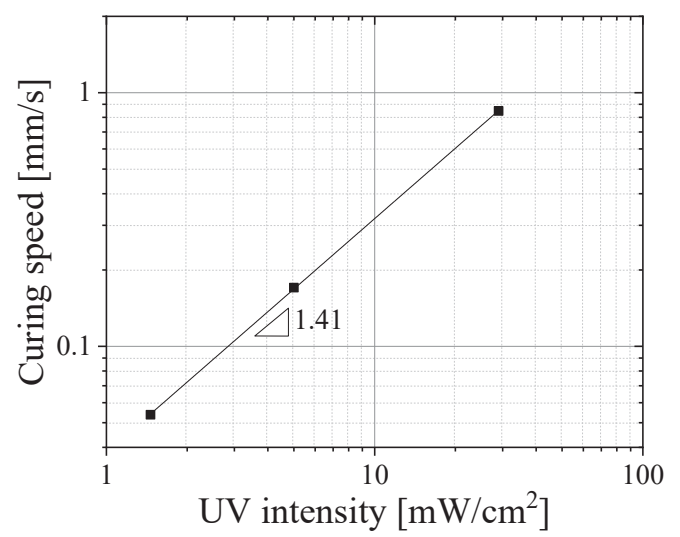

Fig. 6. Scaling relationship between the curing speed and the UV intensity.

Table 1. Linear correlations of onset time.

\begin{tabular}{lcc}
\hline UV intensity & \multicolumn{2}{c}{ Onset time [s] } \\
\cline { 2 - 3 } $\mathrm{mW} / \mathrm{cm}^{2}$ & $\begin{array}{r}\text { Slope } \\
{[\mathrm{s} / \mathrm{mm}]}\end{array}$ & Intercept $[\mathrm{s}]$ \\
\hline 1.46 & 18.6 & 2.01 \\
5 & 5.86 & 1.00 \\
29 & 1.18 & 0.35 \\
\hline
\end{tabular}


The physical meaning of the curing speed is the length to be cured per second. Higher UV intensities supply more photons, which means that photopolymerization propagates faster.

Figure 7 shows the relationships between the normal force (90 s after the UV irradiation stopped), gap width, and UV intensity. The normal force and gap showed a scaling (log-log) correlation. The correlation line was drawn with the normal force data of $5 \mathrm{~mW} / \mathrm{cm}^{2}$. The scaling factor was 1.6 . In general, larger gaps exhibited a larger residual force. The UV intensity dependence is interesting. While the five points on the left hand side of Fig. 7 at 1.46 $\mathrm{mW} / \mathrm{cm}^{2}$ follow the correlation line, the two left points (of which the thicknesses are 0.7 and $0.9 \mathrm{~mm}$ ) deviate. At low UV intensity and large thickness values, sufficient UV intensity could not be delivered to complete the photopolymerization. Moreover, it seems that the two points on the right hand side of $29 \mathrm{~mW} / \mathrm{cm}^{2}$ deviate from the correlation line. These two points indicate that the normal force increased more than expected by the correlation line. It may be because the autoacceleration occurred where the heat of polymerization increased the sample temperature and enhanced the polymerization, at a high UV intensity and for a thick sample.

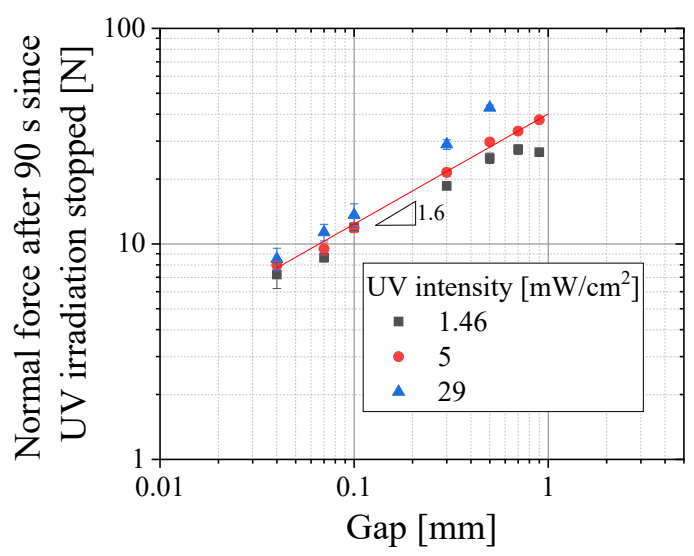

Fig. 7. Relationships between the residual normal force, gap width, and UV intensity.

\section{Conclusion}

The residual force of UV-curing was investigated by measuring the normal force of UV-curable resins between two plates. The normal force increased with increasing UV intensity, and with increasing gap width. The curing onset time became faster for smaller gaps and under higher UV intensities. The curing speed, which is the inverse of the slope of the onset time to the gap width, increased with increasing UV intensity, as expected. The normal force $90 \mathrm{~s}$ after the UV irradiation stopped showed a scaling relationship with the gap width.

In most industrial UV-curing processes, the gap or coating thickness of the resin is less than $0.1 \mathrm{~mm}$. The fact that the residual normal force has a scaling relationship, and that its factor is 1.6, is informative for applications in the precise micro-optics and electronics industries. The developed method is limited to a minimum gap of $10 \mu \mathrm{m}$. If the gap is less than $10 \mu \mathrm{m}$, the measurement was not stabilized. The measurement of normal force for gaps lower than $10 \mu \mathrm{m}$ is a challenge for which we have to build a suitable device. The results of the correlation between normal force, curing speed, and gap width will be utilized as the physical properties for FEA modelling of residual force distribution in UVcuring simulations in our future study.

\section{Acknowledgements}

A part of this study was supported by JSPS KAKENHI for Scientific Research on Innovative Areas "MFS Materials Science" (grant number JP18H05483) and JSPS KAKENHI grant (numbers 16K14469 and 18K04823). We would like to thank Editage (www.editage.jp) for English language editing.

\section{References}

1. B. A. M. Venhoven, A. J. de Gee, and C. L. Davidson, Biomaterials, 14 (1993) 871.

2. H. Lu, J. W. Stansbury, and C. N. Bowman, Dent. Mater., 20 (2004) 979.

3. J. W. Stansbury, M. Trujillo-Lemon, H. Lu, X. Ding, Y. Lin, and J. Ge, Dent. Mater, 21 (2005) 56.

4. R. Suzuki, N. Sakai, A. Sekiguchi, Y. Matsumoto, R. Tanaka, and Y. Hirai, J. Photopolym. Sci. Technol., 23 (2010) 51.

5. C. Gorsche, R. Harikrishna, S. Baudis, P. Knaack, B. Husar, J. Laeuger, H. Hoffmann, and R. Liska, Anal. Chem., 89 (2017) 4958.

6. G. Peer, P. Dorfinger, T. Koch, J. Stampfl, C. Gorsche, and R. Liska, Macromolecules, 51 (2018) 9344.

7. E. Zerobin, M. Markovic, Z. Tomášiková, X.-H. Qin, D. Ret, P. Steinbauer, J. Kitzmüller, W. Steiger, P. Gruber, A. Ovsianikov, R. Liska, and S. Baudis, J. Polym. Sci., 58 (2020) 1288.

8. C. Gorsche, K. Seidler, R. Harikrishna, M. Kury, T. Koch, N. Moszner, and R. Liska, Polymer, 158 (2018) 149.

9. S. Schoerpf, Y. Catel, N. Moszner, C. Gorsche, and R. Liska, Polym. Chem., 10 (2019) 1357. 
10. S. Orman, C. Hofstetter, A. Aksu, F. Reinauer, R. Liska, and S. Baudis, J. Polym. Sci. A: Polym. Chem., 57 (2019) 110.

11. G. Peer, A. Eibel, C. Gorsche, Y. Catel, G. Gescheidt, N. Moszner, and R. Liska, Macromolecules, 52 (2019) 2691.

12. K. Taki and R. Yamada, J. Photopolym. Sci. Technol., 31 (2018) 497.

13. Q. Wang, H. Hiroshima, and H. Atobe, $J$. Photopolym. Sci. Technol., 23 (2010) 33.

14. N. Fujii, T. Tanabe, T. Hirasawa, H. Kawata, N. Sakai, and Y. Hirai, J. Photopolym. Sci. Technol., 22 (2009) 181.

15. Y. Hirai, J. Photopolym. Sci. Technol., 23 (2010)
25.

16. M. Shibata, A. Horiba, Y. Nagaoka, H. Kawata, M. Yasuda, and Y. Hirai, J. Vac. Sci. Technol. B, 28 (2010) C6M108.

17. M. Koyama, M. Shirai, H. Kawata, Y. Hirai, and M. Yasuda, J. Vac. Sci. Technol. B, 35 (2017).

18. T. Tochino, T. Shiotsu, K. Uemura, M. Yasuda, H. Kawata, and Y. Hirai, J. Vac. Sci. Technol. B, 32 (2014).

19. Q. Wang, J. Zhang, R. Zhang, and X. Zheng, J. Photopolym. Sci. Technol., 30 (2017) 545.

20. Y.-M. Huang and C.-P. Jiang, J. Mater. Process. Technol., 159 (2005) 257. 